\title{
Decreased contrast enhancement on high-resolution vessel wall imaging of unruptured intracranial aneurysms in patients taking aspirin
}

\author{
Jorge A. Roa, MD, ${ }^{1,2}$ Mario Zanaty, MD, ${ }^{2}$ Daizo Ishii, MD, ${ }^{2}$ Yongjun Lu, PhD, ${ }^{2}$ David K. Kung, MD, ${ }^{3}$ \\ Robert M. Starke, MD, ${ }^{4}$ James C. Torner, PhD, ${ }^{5}$ Pascal M. Jabbour, MD, ${ }^{6}$ \\ Edgar A. Samaniego, MD, MS, ${ }^{1,2,7}$ and David M. Hasan, MD2
}

Departments of ${ }^{1}$ Neurology and ${ }^{2}$ Neurosurgery, University of lowa Hospitals and Clinics, lowa City, lowa; ${ }^{3}$ Department of Neurosurgery, University of Pennsylvania, Philadelphia, Pennsylvania; ${ }^{4}$ Department of Neurosurgery and Radiology, University of Miami, Florida; ${ }^{5}$ Department of Biostatistics and Epidemiology, University of lowa Hospitals and Clinics, lowa City, lowa; ${ }^{6}$ Department of Neurosurgery, Thomas Jefferson University Hospitals, Philadelphia, Pennsylvania; and ${ }^{7}$ Department of Radiology, University of lowa Hospitals and Clinics, lowa City, lowa

OBJECTIVE Inflammation plays an integral role in the formation, growth, and progression to rupture of unruptured intracranial aneurysms (UIAs). Animal and human studies have suggested that, due to its antiinflammatory effect, aspirin (ASA) may decrease the risks of growth and rupture of UIAs. High-resolution vessel wall imaging (HR-VWI) has emerged as a noninvasive method to assess vessel wall inflammation and UIA instability. To the authors' knowledge, to date no studies have found a significant correlation between patient use of ASA and contrast enhancement of UIAs on HR-VWI.

METHODS The University of lowa HR-VWI Project database was analyzed. This database is a compilation of data on patients with UIAs who prospectively underwent HR-VWI on a 3T Siemens MRI scanner. The presence of aneurysmal wall enhancement was objectively defined using the aneurysm-to-pituitary stalk contrast ratio $\left(\mathrm{CR}_{\text {stalk }}\right)$. This ratio was calculated by measuring the maximal signal intensity in the aneurysmal wall and the pituitary stalk on postcontrast T1weighted images. Data on aneurysm size, morphology, and location and patient demographics and comorbidities were collected. Use of ASA was defined as daily intake of $\geq 81 \mathrm{mg}$ during the previous 6 months or longer. Univariate and multivariate logistic regression analyses were performed to determine factors independently associated with increased contrast enhancement of UIAs on HR-VWI.

RESULTS In total, 74 patients harboring 96 UIAs were included in the study. The mean patient age was $64.7 \pm 12.4$ years, and 60 patients (81\%) were women. Multivariate analysis showed that age (OR 1.12, 95\% Cl 1.05-1.19), aneurysm size $\geq 7 \mathrm{~mm}$ (OR 21.3,95\% Cl 4.88-92.8), and location in the anterior communicating, posterior communicating, and basilar arteries (OR 10.7, 95\% Cl 2.45-46.5) were significantly associated with increased wall enhancement on HR-VWI. On the other hand, use of ASA was significantly associated with decreased aneurysmal wall enhancement on HR-VWI (OR 0.22, 95\% Cl 0.06-0.83, $p=0.026$ ).

CONCLUSIONS The study results establish a correlation between use of ASA daily for $\geq 6$ months and significant decreases in wall enhancement of UIAs on HR-VWI. The findings also demonstrate that detection of wall enhancement using HR-MRI may be a valuable noninvasive method for assessing aneurysmal wall inflammation and UIA instability.

https://thejns.org/doi/abs/10.3171/2019.12.JNS193023

KEYWORDS aspirin; inflammation; high-resolution vessel wall imaging; magnetic resonance imaging; aneurysm; circumferential enhancement; vascular disorders

\begin{abstract}
ABBREVIATIONS $\mathrm{ACA}=$ anterior cerebral artery; $\mathrm{ACOA}=$ anterior communicating artery; $\mathrm{ASA}=$ aspirin; aSAH = aneurysmal SAH; $\mathrm{BA}=$ basilar artery; $\mathrm{CE}-\mathrm{MRA}=\mathrm{contrast}-$ enhanced magnetic resonance angiography; $\mathrm{CR}_{\text {stalk }}=$ aneurysm-to-pituitary stalk contrast ratio; $\mathrm{HR}$-VWI = high-resolution vessel wall imaging; ICA = internal carotid artery; ISUIA = International Study of Unruptured Intracranial Aneurysms; MCA = middle cerebral artery; PCoA = posterior communicating artery; PHASES = population, hypertension, age, size of aneurysm, earlier SAH from another aneurysm, and site of aneurysm; ROI = region of interest; SAH = subarachnoid hemorrhage; SCA = superior cerebellar artery; $\mathrm{SI}=$ signal intensity; UIA = unruptured intracranial aneurysm; VA = vertebral artery.
\end{abstract}

SUBMITTED November 6, 2019. ACCEPTED December 30, 2019.

INCLUDE WHEN CITING Published online March 6, 2020; DOI: 10.3171/2019.12.JNS193023. 
$\mathrm{U}$ NRUPTURED intracranial aneurysms (UIAs) continue to pose a therapeutic dilemma in which the riskbenefit analysis of therapeutic interventions has to be balanced against the natural history of the disease. UIAs are found in $3 \%-5 \%$ of the adult population worldwide. ${ }^{36}$ Although the large majority will never rupture, $27 \%-44 \%$ of patients who develop aneurysmal subarachnoid hemorrhage (aSAH) may die within 12 months, ${ }^{10,24}$ and those who survive experience a $17 \%$ excess mortality after 20 years compared with the general population. ${ }^{23} \mathrm{Re}-$ sults from large prospective trials such as the International Study of Unruptured Intracranial Aneurysms (ISUIA), ${ }^{39}$ the Unruptured Cerebral Aneurysms Study (UCAS), ${ }^{28}$ and the PHASES (population, hypertension, age, size of aneurysm, earlier SAH from another aneurysm, and site of aneurysm) study ${ }^{12}$ have identified demographics (age, sex, and smoking), history of previous aSAH, and aneurysm size, location, and morphology as important determinants of rupture risk.

Currently, it is unclear what prompts the growth and rupture of cerebral aneurysms, but inflammation of the arterial wall may have a pivotal role in this process. ${ }^{5,15,18,20}$ Results from studies of vessel blood flow and histology have led to the proposal that increased hemodynamic stress triggers infiltration of inflammatory cells (mostly M1 macrophages) into the arterial wall. Locally, these cells induce synthesis of cytokines, adhesion molecules, and reactive oxygen species, inflammatory mediators that ultimately lead to endothelial dysfunction, proteolytic destruction of the vascular extracellular matrix by metalloproteinases, and weakening of the aneurysmal wall. Several animal and human studies have shown that aspirin (ASA) attenuates inflammation in the UIA wall and decreases both growth and rupture of UIAs. ${ }^{4,6,13,18,25,34}$

High-resolution vessel wall imaging (HR-VWI) has also been used to study the structure, thickness, and enhancement of the wall of brain aneurysms. Recently, a histological analysis of human aneurysmal wall tissue suggested a significant correlation between increased enhancement and inflammation and, therefore, aneurysm instability. ${ }^{32}$ To date, the only longitudinal study that has prospectively assessed the association between aneurysmal wall enhancement on HR-VWI and risk of aneurysm growth and rupture in patients with UIAs was published by Vergouwen and colleagues. ${ }^{35}$ Their study included a total of 57 patients harboring 65 UIAs. After a median follow-up of 27 months, growth $(n=2)$ or rupture $(n=2)$ was observed in 4 of 19 enhancing aneurysms, whereas none of 46 nonenhancing UIAs showed growth or progressed to rupture. Statistically, Vergouwen et al. found a mean risk difference of $21 \%$ for enhancing compared with nonenhancing UIAs. ${ }^{35}$

In the current study, we aimed to assess the correlation between increased enhancement of UIAs on HR-VWI and classic risk factors of aneurysm rupture and to define the effects of ASA on the wall enhancement of UIAs.

\section{Methods}

\section{Patient Population and Data Collection}

After obtaining Institutional Review Board approval, we conducted an analysis of data from the University of Iowa HR-VWI Project database (January 2015-August 2019). This database collects data on patients who prospectively underwent HR-VWI at diagnosis as part of our institutional protocol to assess incidental UIAs. Nonsaccular aneurysms were excluded from the study. Demographics and clinical information, including patient age, sex, and comorbidities, were gathered from electronic medical records. Patients who were actively smoking or had quit within the last 6 months were categorized as positive for smoking. Use of ASA was defined as daily intake of $\geq 81 \mathrm{mg}$ during the previous 6 months or longer. These cutoffs were based on previous HR-VWI studies published by our group in which we assessed the enhancement of the walls of UIAs with the use of ferumoxytol as a contrast agent. ${ }^{15,17}$

\section{Imaging Acquisition}

Images were routinely acquired with a 3T Siemens MRI scanner (Siemens MAGNETOM Skyra). Our HR-VWI protocol included a 3D T1-weighted SPACE fast-spinecho (FSE) and a 3D T2-weighted sequence. Five minutes after intravenous injection of $0.1 \mathrm{mmol} / \mathrm{kg}$ of the gadolinium-based contrast agent gadobutrol (Gadavist, Bayer Pharmaceuticals), postcontrast 3D T1-weighted SPACE FSE sequence and contrast-enhanced magnetic resonance angiography (CE-MRA) were performed. Technical parameters used for imaging acquisition are described in Supplemental Table 1.

\section{HR-VWI Assessment}

All images were analyzed using our manual segmentation protocol in PACS (Carestream Vue). ${ }^{31}$ Aneurysm size (diameter and neck) was measured based on CE-MRA images. After 6-fold magnification and autocorrection of viewer windowing, the aneurysm was manually coregistered in both pre- and postcontrast T1-weighted sequences using the same slide in all 3 planes (axial, coronal, and sagittal). A biplanar region of interest (ROI) covering the aneurysmal wall was drawn at the level of maximal aneurysm diameter. CE-MRA images were used as a reference to correctly delineate the inner surface of the aneurysmal wall, whereas T2-weighted sequences were used to distinguish the presence of cisterns/CSF surrounding the vessel and define its outer surface. The precontrast T1-weighted sequence was used as a reference to define the overall thickness of the aneurysmal wall and avoid including into the ROI areas of pseudoenhancement secondary to luminal contrast stagnation.

The aneurysm-to-pituitary stalk ratio $\left(\mathrm{CR}_{\text {stalk }}\right)$ was then calculated as the contrast ratio of the maximal signal intensity (SI) of the aneurysmal wall on the postcontrast T1weighted sequence over the averaged pituitary stalk enhancement as described elsewhere. ${ }^{29,30}$ Briefly, 4 different SI points were randomly sampled throughout the pituitary stalk in the sagittal postcontrast T1-weighted images. The maximal SI of all these points was used as the denominator to calculate the $\mathrm{CR}_{\text {stalk }}$ as follows: maximal $\mathrm{SI}_{\text {wall post }} /$ maximal $\mathrm{SI}_{\text {stalk post }}$, where "post" indicates postcontrast. Based on our previous validation study, ${ }^{31}$ a cutoff of $\geq 0.60$ 
for the $\mathrm{CR}_{\text {stalk }}$ was used to define the presence or absence of increased enhancement in the aneurysmal wall.

\section{Statistical Analysis}

Data are summarized as mean \pm SD or mean (range) for continuous variables and as frequency for categorical variables. Patients on regular use of ASA were compared with patients who were not using or were inconsistently using ASA. Aneurysm enhancement on HR-VWI was treated as a binary categorical variable, presence or absence, based on $\mathrm{CR}_{\text {stalk }}$ results. Univariate analysis was used to test baseline associations of covariates with aneurysm enhancement. Statistical analyses of categorical variables were performed using the chi-square and Fisher exact tests; comparison of means was performed using the Student t-test. Factors associated with aneurysm enhancement in the univariate analysis $(\mathrm{p}<0.20)$ were also entered into an aneurysm-specific multivariate logistic regression analysis that was adjusted for aneurysm multiplicity. In the multivariate model, $\mathrm{p}$ values $<0.05$ were considered statistically significant. Odds ratios were estimated for magnitude of effect with 95\% CIs. All statistical analyses were performed with IBM SPSS Statistics version 25.0 (IBM Corp.).

\section{Results \\ Patient Characteristics}

A total of 74 patients with 96 UIAs were included. The mean patient age was $64.7 \pm 12.4$ years, and 60 patients (81\%) were women. Baseline characteristics of the sample are detailed in Table 1. Among comorbidities, 41 patients (55.4\%) had hypertension, 40 (54.1\%) had positive smoking status according to our criteria, and 32 (43.2\%) had hyperlipidemia. Thirty-nine (52.7\%) patients were taking $\geq 81 \mathrm{mg}$ of ASA daily during the previous 6 months or longer. At diagnosis, no patients were on dual antiplatelet therapy. Seven patients $(9.5 \%)$ had a family history of intracranial aneurysms and 4 (5.4\%) had a history of previous aSAH. Additionally, because they were treated promptly, no patients with aneurysmal wall enhancement in this cohort went on to have aSAH.

\section{Aneurysm Characteristics}

The mean aneurysm diameter was $8.1 \mathrm{~mm}$ (range 3-27 $\mathrm{mm})$. Twenty-six $(27.1 \%)$ aneurysms were located in the middle cerebral artery (MCA; 21 MCA bifurcation, $2 \mathrm{M}_{1}$ segment, and $3 \mathrm{M}_{2}$ segment), 25 (26\%) in the internal carotid artery (ICA; 7 ophthalmic/paraophthalmic, 6 supraclinoid, 4 T-terminus, 6 cavernous, and 2 paraclinoid), 15 (14.6\%) in the basilar artery (BA; 11 BA tip, 2 mid-BA, and 2 vertebrobasilar junction), 16 in the anterior communicating artery (ACoA), 5 in the posterior communicating artery $(\mathrm{PCoA}), 5$ in the anterior cerebral artery (ACA; 2 $\mathrm{A}_{1}$ segment, $3 \mathrm{~A}_{2}$ /pericallosal), 3 in the superior cerebellar artery (SCA), and 1 in the vertebral artery (VA). Correlation statistics demonstrated high agreement for areas covered by ROIs in the axial, coronal, and sagittal projections for coregistered pre- and postcontrast T1-weighted images (Pearson coefficients $>0.93, \mathrm{p}<0.001$ ).
TABLE 1. Baseline characteristics of the study sample

\begin{tabular}{|c|c|}
\hline Variable & Value \\
\hline Total no. of patients & 74 \\
\hline Total no. of aneurysms & 96 \\
\hline Mean age in yrs & $64.7 \pm 12.4$ \\
\hline Female sex & $60(81)$ \\
\hline Smoking & $40(54.1)$ \\
\hline Hypertension & $41(55.4)$ \\
\hline Diabetes & $12(16.2)$ \\
\hline Hyperlipidemia & $32(43.2)$ \\
\hline Family history of intracranial aneurysms & $7(9.5)$ \\
\hline History of previous aSAH & $4(5.4)$ \\
\hline $\mathrm{ASA}^{*}$ & $39(52.7)$ \\
\hline Mean aneurysm size in $\mathrm{mm}$ & $8.1(3-27)$ \\
\hline$\geq 7 \mathrm{~mm}$ & $48(50.0)$ \\
\hline$<7 \mathrm{~mm}$ & $48(50.0)$ \\
\hline \multicolumn{2}{|l|}{ Aneurysm location } \\
\hline ICA & $25(26.0)$ \\
\hline $\mathrm{ACoA}$ & $16(16.7)$ \\
\hline ACA & $5(5.2)$ \\
\hline MCA & $26(27.1)$ \\
\hline PCoA & $5(5.2)$ \\
\hline BA & $15(15.6)$ \\
\hline SCA & $3(3.1)$ \\
\hline VA & $1(1.0)$ \\
\hline \multicolumn{2}{|l|}{ Aneurysmal wall enhancement† } \\
\hline Positive & $59(61.5)$ \\
\hline Negative & $37(38.5)$ \\
\hline
\end{tabular}

Values are presented as number of patients (\%) or number of aneurysms (\%) unless otherwise indicated. Continuous values are presented as mean $\pm S D$ or mean (range).

* Daily intake of $\geq 81 \mathrm{mg}$ of ASA during the previous 6 months.

$\dagger$ Defined as positive if $\mathrm{CR}_{\text {stalk }} \geq 0.60$.

\section{Factors Associated With Aneurysmal Wall Enhancement}

Results from univariate and multivariate analyses using $\mathrm{CR}_{\text {stalk }} \geq 0.60$ as the cutoff to define aneurysm enhancement are presented in Table 2. Although odds ratios (ORs) for sex, hypertension, hyperlipidemia, smoking status, and history of previous aSAH favored the presence of wall enhancement on HR-VWI, such associations were statistically nonsignificant $(p>0.20)$ and were not included in the multivariate analysis. Based on the univariate analysis, age; aneurysm location in the ACoA, PCoA, or BA; and aneurysm size $\geq 7 \mathrm{~mm}$ were associated with increased wall enhancement (age OR 1.05, p = 0.007; location OR 2.22, $\mathrm{p}=0.07$; size OR 6.15, $\mathrm{p}<0.001$ ), while ASA use was associated with a lower chance of aneurysmal wall enhancement (OR 0.46, $\mathrm{p}=0.17$ ).

When multivariate analysis was applied, independent factors significantly associated with increased wall enhancement on HR-VWI were age (OR 1.12, 95\% CI 1.05$1.19, \mathrm{p}=0.001)$; location in the ACoA, PCoA, or BA (OR $10.7,95 \%$ CI $2.45-46.5, \mathrm{p}=0.002)$; and size $\geq 7 \mathrm{~mm}(\mathrm{OR}$ 
TABLE 2. Univariate and multivariate analyses using $C R_{\text {stalk }} \geq 0.60$ as the cutoff for aneurysm enhancement

\begin{tabular}{|c|c|c|c|c|}
\hline \multirow[b]{2}{*}{ Variable } & \multicolumn{2}{|c|}{ Univariate } & \multicolumn{2}{|c|}{ Multivariate } \\
\hline & OR $(95 \% \mathrm{Cl})$ & $p$ Value & OR $(95 \% \mathrm{Cl})$ & p Value \\
\hline Age & $1.05(1.01-1.10)$ & 0.007 & $1.12(1.05-1.19)$ & 0.001 \\
\hline Female sex & $1.67(0.48-5.78)$ & 0.42 & - & - \\
\hline Hypertension & $1.27(0.55-2.98)$ & 0.57 & - & - \\
\hline Hyperlipidemia & $1.22(0.53-2.83)$ & 0.65 & - & - \\
\hline Smoking & $1.36(0.59-3.19)$ & 0.47 & - & - \\
\hline History of previous aSAH & $2.54(0.40-16.03)$ & 0.32 & - & - \\
\hline $\mathrm{ASA}^{*}$ & $0.46(0.15-1.36)$ & 0.17 & $0.22(0.06-0.83)$ & 0.026 \\
\hline Aneurysm size $\geq 7 \mathrm{~mm}$ & $6.15(2.42-15.65)$ & $<0.001$ & $21.3(4.88-92.8)$ & $<0.001$ \\
\hline Aneurysm location† & $2.22(0.94-5.24)$ & 0.07 & $10.7(2.45-46.5)$ & 0.002 \\
\hline
\end{tabular}

* Daily intake of $\geq 81 \mathrm{mg}$ ASA during the last 6 months.

$\dagger$ Refers to locations in the ACOA, PCoA, and BA.

21.3, 95\% CI 4.88-92.8, $\mathrm{p}<0.001)$. On the other hand, daily intake of ASA was significantly protective (OR 0.22 , 95\% CI $0.06-0.83, \mathrm{p}=0.026$ ), reducing the odds of aneurysmal wall enhancement by an average of $78 \%$.

\section{Discussion}

\section{Aneurysm Enhancement on HR-VWI and Instability}

This study is to our knowledge the first to establish a significant correlation between ASA intake and decreased wall enhancement of UIAs. Several studies have evaluated the relationship between wall enhancement on HRVWI and classic risk factors of aneurysm rupture. Backes et al. ${ }^{3}$ showed that the strongest determinant of wall enhancement in 89 predominantly small UIAs $(84 \%<7$ $\mathrm{mm}$ ) was aneurysm size (OR 14.8, 95\% CI 2.1-104.6 for $\geq 7.0 \mathrm{~mm}$ ), followed by location in the PCoA (OR 3.6, 95\% CI 1.1-11.4) or MCA (OR 3.0, 95\% CI 1.0-8.6). No association was found between aneurysmal wall enhancement and smoking status, use of statins, hypertension, or irregular shape. ${ }^{3}$ Liu et al. ${ }^{26}$ also found that aneurysm size was independently associated with aneurysmal wall enhancement in 48 patients with 61 predominantly large UIAs $(59 \% \geq 7 \mathrm{~mm})$. Lv et al. ${ }^{27}$ demonstrated a significant association between HR-VWI enhancement, aneurysm size $\geq 7 \mathrm{~mm}$, and location in the ACA, PCoA, and posterior circulation arteries $(\mathrm{p}<0.001)$. Wang and colleagues ${ }^{37}$ analyzed 88 UIAs and demonstrated that irregular shape and high depth/neck width aspect ratio were significantly associated with wall enhancement (OR 12.5, $\mathrm{p}=0.02$, and OR $32.9, \mathrm{p}=0.01$, respectively). Hartman et al. compared wall enhancement of 65 UIAs and their PHASES scores, showing that aneurysms with PHASES scores of $>3$ were more likely to demonstrate wall enhancement $(42.1 \%$ vs $14.8 \%, \mathrm{p}=0.022) .{ }^{14}$ Recently, Vergouwen and colleagues prospectively followed 57 patients with 65 UIAs to assess the association between aneurysmal wall enhancement and instability. ${ }^{35}$ After a median follow-up period of 27 months, 4/19 enhancing aneurysms showed instability (growth in 2, rupture in 2), whereas none of 46 nonenhancing UIAs demonstrated significant changes (risk difference $21 \%, 95 \%$ CI 3\%-39\%). ${ }^{35}$ These findings suggested that gadolinium wall enhancement of UIAs is associated with increased risk of instability and might be used as an independent predictor of aneurysm growth and rupture.

Although they produced interesting findings, all of these studies relied on subjective assessments and arbitrary scales to determine the presence or absence of aneurysm enhancement on HR-VWI. In 2016, Omodaka et al. ${ }^{30}$ proposed the use of the $\mathrm{CR}_{\text {stalk }}$ on postcontrast T1weighted imaging as a standardized tool to objectively quantify contrast enhancement in the wall of UIAs on HRVWI. Using maximal SI values, the authors reported that a $\mathrm{CR}_{\text {stalk }}$ cutoff of $\geq 0.64$ achieved sensitivity of $75 \%$ and specificity of $83 \%$ for distinguishing ruptured aneurysms $(n=28)$ from UIAs $(n=76)$. Later on, the same group compared the $\mathrm{CR}_{\text {stalk }}$ in 69 stable UIAs, 26 evolving UIAs, and 67 ruptured aneurysms and reported that evolving UIAs had significantly higher $\mathrm{CR}_{\text {stalk }}$ values than stable UIAs (0.54 vs $0.34, p<0.0001)$, but lower $\mathrm{CR}_{\text {stalk }}$ values than ruptured aneurysms $(0.54$ vs $0.83, \mathrm{p}<0.0002) .{ }^{29} \mathrm{An}$ internal validation study performed by our group compared several objective methodologies to quantify SI in the wall of UIAs using HR-VWI. This analysis showed that the $\mathrm{CR}_{\text {stalk }}$ calculated with maximal SI achieved the best area under the curve among all contrast ratios, and a cutoff $\geq 0.60$ reached an optimal sensitivity of $81.5 \%$ and specificity of $60.1 \% \cdot{ }^{31}$ Consequently, we used maximal SI values to calculate the $\mathrm{CR}_{\text {stalk }}$ as a determinant of aneurysmal wall enhancement in this cohort.

\section{HR-VWI and Aneurysm Inflammation}

Previous studies have demonstrated that it is possible to image active wall inflammation of UIAs in vivo. ${ }^{15,17}$ Our group has investigated the potential use of targeted imaging with ferumoxytol-enhanced MRI (Fe-MRI) to assess the effects of ASA on UIA vessel wall inflammation. Since ferumoxytol is a superparamagnetic iron oxide agent phagocytized by macrophages and cleared 24 to 72 hours after injection, it can be used as a contrast agent to target active inflammatory cells in MRI. In a prospective 
study of 11 patients with UIAs randomized into an ASAtreated $(n=6)$ or no-ASA $(n=5)$ group, we found a significant decrease in the SI of the aneurysmal wall of patients on ASA after 3 months. ${ }^{17}$ Moreover, immunostaining of histological samples following microsurgical aneurysm clipping showed a significant decrease in the number of inflammatory cells and expression of cytokines in the aneurysmal wall. ${ }^{17}$ These findings indicated that ASA effectively reduces inflammation in the aneurysmal wall and Fe-MRI detects these changes in an early phase.

Gadolinium-enhanced HR-VWI studies have also suggested a significant correlation between aneurysmal wall enhancement, inflammatory changes, and instability. After analyzing 10 postclipping wall specimens from UIAs with presurgical HR-VWI available, our group demonstrated that aneurysms with avid enhancement had higher average atherosclerotic wall thickness $(\mathrm{p}=0.003)$, macrophage infiltration, and cellularity $(\mathrm{p}=0.048)$ than aneurysms with mildly enhancing or no-enhancing walls. ${ }^{22}$ Similarly, Shimonaga et al. ${ }^{33}$ found a significant association between aneurysmal wall enhancement and the presence of vasa vasorum disease, neovascularization, and macrophage infiltration on histological analysis of 9 UIAs. A critical review of the current evidence of vessel wall inflammation in enhancing UIAs can be found elsewhere. ${ }^{32}$

\section{ASA and Aneurysm Inflammation}

Several preclinical studies have suggested that ASA beneficially attenuates the aberrant inflammatory microenvironment within the aneurysmal wall, ultimately preventing rupture. Aoki et al. ${ }^{1,2}$ hypothesized that the COX-2 (cyclooxygenase)/mPGES (microsomal prostaglandin E2 synthase-1)/PGE-2 (prostaglandin E2)/E2 receptor 4 pathway could be involved in aneurysm pathogenesis and progression. A mouse study conducted by our group comparing $\mathrm{mPGES}$-deleted versus wild-type animals suggested that ASA, via its inhibitory effect over COX-2 activity, effectively attenuates the proinflammatory environment in mPGES-1-deficient mice by decreasing the concentration of upstream mPGES-1 substrates ${ }^{16}$ Furthermore, the antiinflammatory response to ASA may differ in males versus females. Chalouhi et al. ${ }^{8}$ observed that male mice on ASA exhibit higher levels of 15-hydroxyprostaglandin dehydrogenase (15-PGDH; which catalyzes the formation of antiinflammatory 15-keto-PGE-2) than female mice receiving ASA. After administration of a 15-PGDH activator to female mice on ASA, the rate of aneurysm rupture was similar to the rate in male mice receiving ASA only? Thus, decreased concentrations of 15-PGDH in women may not only explain their higher propensity to aneurysm formation and rupture, but may also account for a decreased antiinflammatory effect of ASA in the wall of UIAs harbored by these patients. We validated these findings in a human study quantifying levels of 15-PGDH in the aneurysmal lumen.?

Clinical studies in humans have also shown significant associations between ASA use and reduced risk of aSAH. ${ }^{21}$ Our group performed a nested case-control study on patients selected from the prospective cohort of 1691 patients with untreated UIAs from the ISUIA. After categorizing these patients into ASA usage frequency groups, we found that patients who used ASA at least 3 times per week had decreased odds of aneurysmal hemorrhage compared with patients who reported no ASA usage (OR 0.27, 95\% CI 0.11-0.67). ${ }^{18}$ These findings were later confirmed by García-Rodríguez et al. ${ }^{11}$ in a large, rigorous European study (1797 patients with ICH, 1340 with SAH, and 10,000 controls) in which long-term (> 3 years) intake of ASA was associated with decreased odds of SAH (OR 0.63, 95\% CI 0.45-0.90). ASA use was not significantly associated with an increased risk of ICH (OR 0.89, 95\% CI 0.73-1.09). ${ }^{11}$ More recently, a case-control study by Hostettler et al. ${ }^{19}$ of 2334 patients (1729 with aneurysm rupture, 605 with UIAs) showed a significant protective effect of ASA on aneurysm rupture status (OR 0.28, 95\% CI 0.20-0.40).

\section{ASA and Aneurysm Enhancement on HR-VWI}

Our findings of increased wall enhancement in UIAs $\geq 7 \mathrm{~mm}$ located in the ACoA, PCoA, and BA are consistent with the findings in the literature presented here. However, to our knowledge the association between ASA and decreased wall enhancement in patients with UIA has not been previously reported. Although previous evidence failed to support such a correlation, most existing studies using HR-VWI did not establish clear criteria to define ASA use, or simply excluded these patients..$^{14,26,29,35,38}$ It has been widely demonstrated that the antiinflammatory effect of ASA is dependent on the dose, frequency, and duration of the regimen. Can et al. ${ }^{4}$ performed a case-control study with 4701 patients (1284 with ruptured aneurysms, 3404 with UIAs). This study not only found that ASA was associated with decreased odds of aSAH (OR 0.60, 95\% CI $0.53-0.81$ ), it also showed a significant inverse doseresponse relationship between ASA use and aSAH (OR $0.65,95 \%$ CI $0.53-0.81) .{ }^{4}$ In the studies by Edjlali et al. ${ }^{9}$ and Lv et al., ${ }^{27}$ ASA use was only assessed for frequency (daily intake), without considering dose or duration. Although Backes et al. ${ }^{3}$ considered patients with a daily intake of $\geq 80 \mathrm{mg}$ ASA, the minimum duration criterion was only 3 months, which might not be long enough to establish a substantial ASA-mediated antiinflammatory response in the aneurysmal wall detectable using gadolinium-enhanced MRI. Our study used a strict criterion to define ASA use as daily intake of $\geq 81 \mathrm{mg}$ during the previous 6 months or longer.

\section{Limitations}

Our study is limited by its retrospective design, which did not allow us to accurately estimate a dose-response relationship between ASA and aneurysmal wall enhancement. Statistically, the small sample size also decreases the power of the study, with wide $95 \%$ CIs for some of the associated variables. However, imaging data in this cohort were collected systematically, with prospective follow-up. Our findings result from a homogenous sample of Caucasian patients with UIAs, and thus may not be generalizable to populations with a different racial background. Although ASA compliance was documented in the medical chart, this may not reflect true compliance. At our institution, patients who have already had an intracranial aneu- 
rysm found incidentally are put on ASA. Other indications for ASA included coronary and peripheral vascular disease. Our multivariable analysis was adjusted for most of these comorbidities (hypertension, hyperlipidemia, history of previous aSAH). Thus, the risk of confounding bias for ASA use in this cohort is very low. However, patients with hypertension and hyperlipidemia could not be stratified into controlled and uncontrolled categories due to heterogeneous information in the medical charts.

Despite the coregistration protocol applied for HRVWI analysis, some locations preclude easy demarcation of the contour of the aneurysmal wall due to surrounding SI artifacts. This is especially difficult for aneurysms located in the paraclinoid, paraophthalmic, and cavernous segments of the ICA (close proximity to the cavernous/ sphenoid sinuses and the dural folds of the skull base), and for those located in the distal MCA ( $\mathrm{M}_{2}$ and $\mathrm{M}_{3}$ segments) surrounded by brain parenchyma. Additionally, our logistic regression analyses assumed a $\mathrm{CR}_{\text {stalk }}$ cutoff of $\geq 0.60$ and the use of maximal SI to objectively define significant contrast enhancement in the aneurysm. Although such assumptions are well supported by previous studies and provide a reliable method for objective assessment of HRVWI, ${ }^{30,31}$ the gold standard would be longitudinal followup of possible growth and/or rupture of UIAs based on follow-up MRI and clinical assessments.

\section{Conclusions}

ASA use is associated with a decreased rate of aneurysmal wall enhancement, whereas UIAs $\geq 7 \mathrm{~mm}$ located in the ACoA, PCoA, and BA demonstrate significantly increased wall enhancement. HR-MRI may be useful as a noninvasive method for assessment of aneurysmal wall inflammation and UIA instability.

\section{Acknowledgments}

This work was conducted at the University of Iowa MR Research Facility on an MRI instrument funded by 1S10RR028821-01.

\section{References}

1. Aoki T, Frösen J, Fukuda M, Bando K, Shioi G, Tsuji K, et al: Prostaglandin E2-EP2-NF- $\mathrm{KB}$ signaling in macrophages as a potential therapeutic target for intracranial aneurysms. Sci Signal 10:eaah6037, 2017

2. Aoki T, Nishimura M, Matsuoka T, Yamamoto K, Furuyashiki T, Kataoka H, et al: PGE(2) -EP(2) signalling in endothelium is activated by haemodynamic stress and induces cerebral aneurysm through an amplifying loop via NF-кB. Br J Pharmacol 163:1237-1249, 2011

3. Backes D, Hendrikse J, van der Schaaf I, Algra A, Lindgren $\mathrm{AE}$, Verweij BH, et al: Determinants of gadolinium-enhancement of the aneurysm wall in unruptured intracranial aneurysms. Neurosurgery 83:719-725, 2018

4. Can A, Rudy RF, Castro VM, Yu S, Dligach D, Finan S, et al: Association between aspirin dose and subarachnoid hemorrhage from saccular aneurysms: a case-control study. Neurology 91:e1175-e1181, 2018

5. Chalouhi N, Ali MS, Jabbour PM, Tjoumakaris SI, Gonzalez LF, Rosenwasser RH, et al: Biology of intracranial aneurysms: role of inflammation. J Cereb Blood Flow Metab 32:1659-1676, 2012
6. Chalouhi N, Atallah E, Jabbour P, Patel PD, Starke RM, Hasan D: Aspirin for the prevention of intracranial aneurysm rupture. Neurosurgery 64 (CN_suppl_1):114-118, 2017

7. Chalouhi N, Jabbour P, Zanaty M, Starke RM, Torner J, Nakagawa D, et al: Sex differential in 15-hydroxyprostaglandin dehydrogenase levels in the lumen of human intracranial aneurysms. J Am Heart Assoc 6:e006639, 2017

8. Chalouhi N, Starke RM, Correa T, Jabbour PM, Zanaty M, Brown RD Jr, et al: Differential sex response to aspirin in decreasing aneurysm rupture in humans and mice. Hypertension 68:411-417, 2016

9. Edjlali M, Gentric JC, Régent-Rodriguez C, Trystram D, Hassen WB, Lion S, et al: Does aneurysmal wall enhancement on vessel wall MRI help to distinguish stable from unstable intracranial aneurysms? Stroke 45:3704-3706, 2014

10. Feigin VL, Lawes CM, Bennett DA, Barker-Collo SL, Parag $\mathrm{V}$ : Worldwide stroke incidence and early case fatality reported in 56 population-based studies: a systematic review. Lancet Neurol 8:355-369, 2009

11. García-Rodríguez LA, Gaist D, Morton J, Cookson C, González-Pérez A: Antithrombotic drugs and risk of hemorrhagic stroke in the general population. Neurology 81:566574, 2013

12. Greving JP, Wermer MJ, Brown RD Jr, Morita A, Juvela S, Yonekura M, et al: Development of the PHASES score for prediction of risk of rupture of intracranial aneurysms: a pooled analysis of six prospective cohort studies. Lancet Neurol 13:59-66, 2014

13. Gross BA, Rosalind Lai PM, Frerichs KU, Du R: Aspirin and aneurysmal subarachnoid hemorrhage. World Neurosurg 82:1127-1130, 2014

14. Hartman JB, Watase H, Sun J, Hippe DS, Kim L, Levitt M, et al: Intracranial aneurysms at higher clinical risk for rupture demonstrate increased wall enhancement and thinning on multicontrast 3D vessel wall MRI. Br J Radiol 92:20180950, 2019

15. Hasan D, Chalouhi N, Jabbour P, Dumont AS, Kung DK, Magnotta VA, et al: Early change in ferumoxytol-enhanced magnetic resonance imaging signal suggests unstable human cerebral aneurysm: a pilot study. Stroke 43:3258-3265, 2012

16. Hasan D, Hashimoto T, Kung D, Macdonald RL, Winn HR, Heistad D: Upregulation of cyclooxygenase-2 (COX-2) and microsomal prostaglandin E2 synthase-1 (mPGES-1) in wall of ruptured human cerebral aneurysms: preliminary results. Stroke 43:1964-1967, 2012

17. Hasan DM, Chalouhi N, Jabbour P, Dumont AS, Kung DK, Magnotta VA, et al: Evidence that acetylsalicylic acid attenuates inflammation in the walls of human cerebral aneurysms: preliminary results. J Am Heart Assoc 2:e000019, 2013

18. Hasan DM, Mahaney KB, Brown RD Jr, Meissner I, Piepgras DG, Huston J, et al: Aspirin as a promising agent for decreasing incidence of cerebral aneurysm rupture. Stroke 42:3156-3162, 2011

19. Hostettler IC, Alg VS, Shahi N, Jichi F, Bonner S, Walsh D, et al: Characteristics of unruptured compared to ruptured intracranial aneurysms: a multicenter case-control study. Neurosurgery 83:43-52, 2018

20. Hudson JS, Hoyne DS, Hasan DM: Inflammation and human cerebral aneurysms: current and future treatment prospects. Future Neurol 8:663-676, 2013

21. Hudson JS, Marincovich AJ, Roa JA, Zanaty M, Samaniego EA, Hasan DM: Aspirin and intracranial aneurysms. Stroke 50:2591-2596, 2019

22. Hudson JS, Zanaty M, Nakagawa D, Kung DK, Jabbour P, Samaniego EA, et al: Magnetic resonance vessel wall imaging in human intracranial aneurysms. Stroke 50:e1, 2019

23. Huhtakangas J, Lehto H, Seppä K, Kivisaari R, Niemelä M, Hernesniemi J, et al: Long-term excess mortality after aneurysmal subarachnoid hemorrhage: patients with multiple aneurysms at risk. Stroke 46:1813-1818, 2015 
24. Karamanakos PN, von Und Zu Fraunberg M, Bendel S, Huttunen T, Kurki M, Hernesniemi J, et al: Risk factors for three phases of 12-month mortality in 1657 patients from a defined population after acute aneurysmal subarachnoid hemorrhage. World Neurosurg 78:631-639, 2012

25. Krings T, Mandell DM, Kiehl TR, Geibprasert S, Tymianski M, Alvarez H, et al: Intracranial aneurysms: from vessel wall pathology to therapeutic approach. Nat Rev Neurol 7:547559,2011

26. Liu P, Qi H, Liu A, Lv X, Jiang Y, Zhao X, et al: Relationship between aneurysm wall enhancement and conventional risk factors in patients with unruptured intracranial aneurysms: a black-blood MRI study. Interv Neuroradiol 22:501-505, 2016

27. Lv N, Karmonik C, Chen S, Wang X, Fang Y, Huang Q, et al: Relationship between aneurysm wall enhancement in vessel wall magnetic resonance imaging and rupture risk of unruptured intracranial aneurysms. Neurosurgery 84:E385-E391, 2019

28. Morita A, Kirino T, Hashi K, Aoki N, Fukuhara S, Hashimoto N, et al: The natural course of unruptured cerebral aneurysms in a Japanese cohort. N Engl J Med 366:2474-2482, 2012

29. Omodaka S, Endo H, Niizuma K, Fujimura M, Inoue T, Endo $\mathrm{T}$, et al: Circumferential wall enhancement in evolving intracranial aneurysms on magnetic resonance vessel wall imaging. J Neurosurg 131:1262-1268, 2019

30. Omodaka S, Endo H, Niizuma K, Fujimura M, Inoue T, Sato $\mathrm{K}$, et al: Quantitative assessment of circumferential enhancement along the wall of cerebral aneurysms using MR imaging. AJNR Am J Neuroradiol 37:1262-1266, 2016

31. Roa JA, Zanaty M, Osorno-Cruz C, Ishii D, Bathla G, Ortega-Gutierrez S, et al: Objective quantification of contrast enhancement of unruptured intracranial aneurysms: a highresolution vessel wall imaging validation study. J Neurosurg [epub ahead of print February 7, 2020. DOI: 10.3171/2019.12. JNS192746]

32. Samaniego EA, Roa JA, Hasan D: Vessel wall imaging in intracranial aneurysms. J Neurointerv Surg 11:1105-1112, 2019

33. Shimonaga K, Matsushige T, Ishii D, Sakamoto S, Hosogai $\mathrm{M}$, Kawasumi T, et al: Clinicopathological insights from vessel wall imaging of unruptured intracranial aneurysms. Stroke 49:2516-2519, 2018

34. Starke RM, Chalouhi N, Ding D, Hasan DM: Potential role of aspirin in the prevention of aneurysmal subarachnoid hemorrhage. Cerebrovasc Dis 39:332-342, 2015

35. Vergouwen MDI, Backes D, van der Schaaf IC, Hendrikse J, Kleinloog R, Algra A, et al: Gadolinium enhancement of the aneurysm wall in unruptured intracranial aneurysms is associated with an increased risk of aneurysm instability: a follow-up study. AJNR Am J Neuroradiol 40:1112-1116, 2019

36. Vlak MH, Algra A, Brandenburg R, Rinkel GJ: Prevalence of unruptured intracranial aneurysms, with emphasis on sex, age, comorbidity, country, and time period: a systematic review and meta-analysis. Lancet Neurol 10:626-636, 2011
37. Wang GX, Li W, Lei S, Ge XD, Yin JB, Zhang D: Relationships between aneurysmal wall enhancement and conventional risk factors in patients with intracranial aneurysm: a high-resolution MRI study. J Neuroradiol 46:25-28, 2019

38. Wang GX, Wen L, Lei S, Ran Q, Yin JB, Gong ZL, et al: Wall enhancement ratio and partial wall enhancement on MRI associated with the rupture of intracranial aneurysms. J Neurointerv Surg 10:566-570, 2018

39. Wiebers DO, Whisnant JP, Huston J III, Meissner I, Brown RD Jr, Piepgras DG, et al: Unruptured intracranial aneurysms: natural history, clinical outcome, and risks of surgical and endovascular treatment. Lancet 362:103-110, 2003

\section{Disclosures}

The research by E.A.S. is funded by the Brain Aneurysm Research Grant from the Bee Foundation, and by the Society of Vascular and Interventional Neurology (SVIN) 2019 grant; he also has consulting agreements with MicroVention and Medtronic. The research by R.M.S. is supported by the NREF, Joe Niekro Foundation, Brain Aneurysm Foundation, Bee Foundation, the National Institutes of Health (UL1TR002736, KL2TR002737) through the Miami Clinical and Translational Science Institute, the National Center for Advancing Translational Sciences, and the National Institute on Minority Health and Health Disparities; he also has consulting and teaching agreements with Penumbra, Abbott, Medtronic, InNeuroCo, and Cerenovus.

\section{Author Contributions}

Conception and design: Hasan, Roa, Samaniego. Acquisition of data: Roa. Analysis and interpretation of data: Roa, Zanaty, Starke. Drafting the article: Roa, Zanaty. Critically revising the article: all authors. Reviewed submitted version of manuscript: Hasan, Roa. Approved the final version of the manuscript on behalf of all authors: Hasan. Statistical analysis: Roa, Zanaty, Torner. Study supervision: Hasan.

\section{Supplemental Information Online-Only Content}

Supplemental material is available with the online version of the article.

Supplemental Table 1. https://thejns.org/doi/suppl/10.3171/ 2019.12.JNS193023.

\section{Correspondence}

David M. Hasan: University of Iowa Hospitals and Clinics, Iowa City, IA.david-hasan@uiowa.edu. 